\title{
Spatio-temporal interfacial potential patterns during the electrocatalyzed oxidation of formic acid on Bi-modified Pt
}

Cite as: J. Chem. Phys. 115, 1485 (2001); https://doi.org/10.1063/1.1379535

Submitted: 28 November 2000 . Accepted: 25 April 2001 . Published Online: 10 July 2001

Jaeyoung Lee, Johannes Christoph, Peter Strasser, Markus Eiswirth, and Gerhard Ertl

ARTICLES YOU MAY BE INTERESTED IN

Edge effects in an electrochemical reaction: $\mathrm{HCOOH}$ oxidation on a Pt ribbon

The Journal of Chemical Physics 126, 144702 (2007); https://doi.org/10.1063/1.2717163

A General Theory of the Reaction Loci in Emulsion Polymerization

The Journal of Chemical Physics 13, 381 (1945); https://doi.org/10.1063/1.1724054

A surface-electrochemical basis for the direct logarithmic growth law for initial stages of extension of anodic oxide films formed at noble metals

The Journal of Chemical Physics 93, 8361 (1990); https://doi.org/10.1063/1.459319
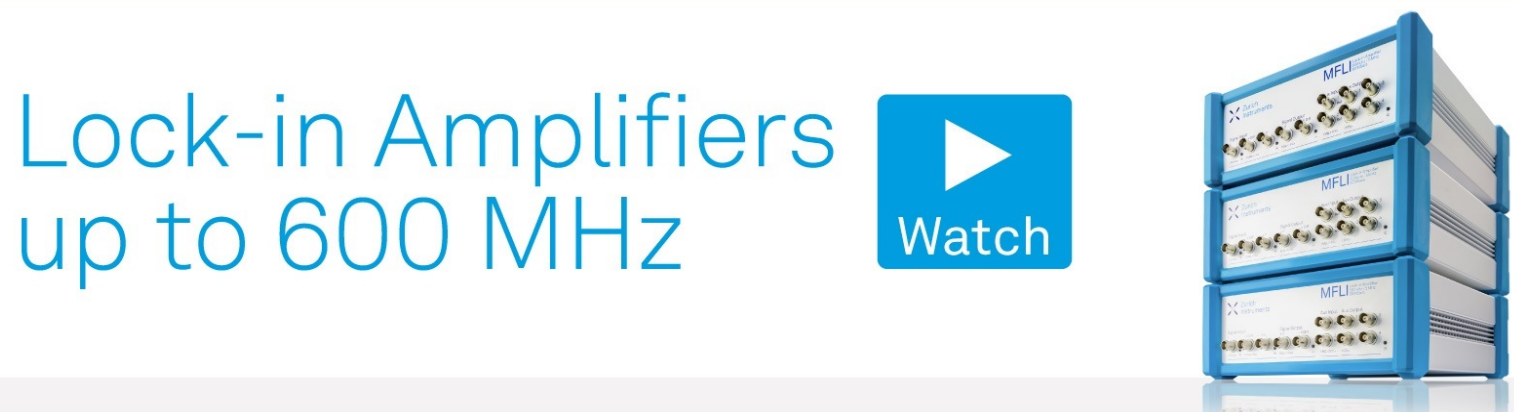

J. Chem. Phys. 115, 1485 (2001); https://doi.org/10.1063/1.1379535

115, 1485

(c) 2001 American Institute of Physics. 


\title{
Spatio-temporal interfacial potential patterns during the electrocatalyzed oxidation of formic acid on Bi-modified Pt
}

\author{
Jaeyoung Lee, Johannes Christoph, Peter Strasser, Markus Eiswirth, ${ }^{\text {a) }}$ and Gerhard Ertl \\ Fritz-Haber-Institut der Max-Planck-Gesellschaft, Faradayweg 4-6, D-14195 Berlin, Germany
}

(Received 28 November 2000; accepted 25 April 2001)

\begin{abstract}
We report experimental observations of the spatio-temporal dynamics in the electro-oxidation of formic acid on a Pt ring electrode modified by bismuth adatoms. Bismuth modification significantly enhanced the current density and it was found to considerably increase the existence range of oscillations and spatio-temporal self-organization. Hidden negative differential resistance and the existence of a Hopf bifurcation were deduced from the electrochemical impedance spectra and the occurrence of galvanostatic oscillations. The pattern formation resulted from hybrid effects of the nonlinear chemistry during formic acid oxidation and the long-range coupling of the interfacial potential induced by the chosen geometry (ring type) of the working electrode. Reversible transitions between traveling pulses and oscillating standing waves were observed when the outer potential or the formic acid concentration near the electrode were used as control parameters. Experimental results were compared with computer simulations of a reaction-migration system. The role of electrode inhomogeneities in pattern formation and the transform between patterns were discussed. (C) 2001 American Institute of Physics. [DOI: 10.1063/1.1379535]
\end{abstract}

\section{INTRODUCTION}

The electrocatalyzed oxidation of small organic molecules such as formic acid (FA), formaldehyde, or methanol, on noble metal electrodes are of interest in connection with anodic reactions in devices for direct electrochemical energy conversion (direct $\mathrm{C}_{1}$ fuel cells). ${ }^{1-7}$ Common to these reactions is the tendency to self-poisoning of the reactive surface, mostly by carbon monoxide, and the existence of parallel reaction channels.

A remarkable feature of these electrocatalytic oxidation reactions is the tendency for formation of self-organized spatio-temporal instabilities at higher overpotentials or current loads. ${ }^{1,3,8-23}$ Dynamical instabilities in electrochemistry most frequently arise through the interplay of the outer electrical load line and a sequence of faradaic processes with nonmonotonous current-potential characteristics. ${ }^{24-27}$ The simplest dynamical instability is a coexistence of two (bistability) or more (multistability) stable stationary operating points for the same value of an outer system constraint (applied outer potential or current). ${ }^{24,25,27}$ More important in the present context, however, are nonstationary operation points in which the measured currents or potentials temporally exhibit sustained periodic behavior, i.e., autonomous electrochemical oscillations. ${ }^{26-28}$

The occurrence of temporally self-organized dynamical states immediately raises the question of concomitant spatial instability, that is, spontaneously generated spatial inhomogeneities in the catalytic activity. However, since the measured electrochemical current or potential represent integral quantities across the interface, the investigation of their relation to the corresponding spatial distribution of the interfacial potential, that is, the local electrocatalytic activity profile

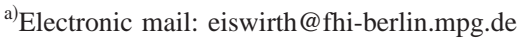

is of importance for a detailed understanding of electrochemical instabilities.

In this work, we present the investigation of spatiotemporal patterns in the interfacial potentials associated with the electro-oxidation of formic acid on a Pt electrode modified by bismuth adatoms. With regard to an earlier study, ${ }^{23}$ it is shown that adatom modifications can significantly enhance the susceptibility of the catalytic interface towards pattern forming instabilities. ${ }^{29,30}$ Of primary interest in the dependence of the interfacial potential distribution on system parameters such as applied potential or mass-transport conditions.

\section{EXPERIMENT}

\section{A. Electrochemical setup}

The principal gas-tight, three-electrode, and onecompartment arrangement for the measurement of local potential distributions at electrochemical interfaces were used. The electrochemical cell body consisted of a glass cylinder capped with a Teflon lid holding all electrodes. A smooth polycrystalline $\mathrm{Pt}$ ring with inner diameter of $34.5 \mathrm{~mm}$ and with outer diameter of $40.5 \mathrm{~mm}$ (thickness: $0.1 \mathrm{~mm}$ ) was used as working electrode (WE). The geometric area of the WE was $7 \mathrm{~cm}^{2}$. A concentric platinized Pt wire ring of 70 $\mathrm{mm}$ diameter (1 $\mathrm{mm}$ wire thickness) was used as counter electrode. The tip of the Luggin-Haber capillary hosting a $\mathrm{Hg} / \mathrm{Hg}_{2} \mathrm{SO}_{4}, \mathrm{~K}_{2} \mathrm{SO}_{4}$, (sat'd) reference electrode was placed in the center of the ring working electrode. Unless stated otherwise, experiments have been executed in the absence of magnetic stirring or forced convection (FC) by nitrogen bubbling.

To measure the instantaneous local interfacial potentials on the Pt ring WE, 11 potential microprobes were equispaced 
(in $30^{\circ}$ angle) along the WE; an additional trigger electrode at the 12 o'clock was not used in this work. Each microprobe consisted of a glass tube with a microcapillary (inner diameter: ca. $200 \mu \mathrm{m}$ ) at one end. The microprobes capped with a commercial $\mathrm{Hg} / \mathrm{Hg}_{2} \mathrm{SO}_{4}$ electrode were filled with a $0.5 \mathrm{M}$ $\mathrm{Na}_{2} \mathrm{SO}_{4}$ solution (Merck, p.a.) and mounted one by one into the Teflon lid followed by careful alignment of all capillary tips with respect to the $\mathrm{Pt}$ ring WE. The distance between WE and the end of the capillary of microprobes was ca. 100-300 $\mu \mathrm{m}$. The spatial positions along the WE as reported in the following experimental data are numbered from 1 to 11 and directly correspond to the respective position of one of the potential probes. All local probe potentials reported hereafter refer to the instantaneous potential difference between the WE and the opening of the tip of the respective microprobe. Neglecting the remaining ohmic potential drop, the probe signals represent a good approximation to the instantaneous local interfacial potentials of the WE.

A home-built potentiostat/scan generator (Electronic Lab, Fritz-Haber-Institut der MPG) was used for all cyclic voltammetry $(\mathrm{CV})$ and chronoamperometry (CA) experiments and the data was transferred to an IBM compatible PC controlled by a GPIB interface. Galvanostatic experiments were performed by means of a bi-potentiostat (EG\&G, Model 366), which can also be used as galvanostat.

\section{B. Solutions and electrode treatment}

All solutions were prepared with ultrapure water (Millipore Milli-Q Water, $18 \mathrm{M} \Omega \mathrm{cm}$ ). Prior to the experiment, the Pt ring WE was first subjected to cleaning with acetone and pure water in an ultrasonic bath followed by chemical cleaning in a mixture of conc. $\mathrm{H}_{2} \mathrm{SO}_{4}$ (Merck, suprapure): $30 \%$ $\mathrm{H}_{2} \mathrm{O}_{2}(1: 1)$. Then a preliminary check of the voltammetric curve between $-600 \mathrm{mV}$ and $+500 \mathrm{mV}$ of the WE was performed in $0.5 \mathrm{M} \mathrm{H}_{2} \mathrm{SO}_{4}$ deaerated by high-purity $\mathrm{N}_{2}$ $(99.999 \%)$ to verify the absence of any residuals of adatoms. For all experiments involving formic acid oxidation, a deaerated solution mixture of $0.1 \mathrm{M} \mathrm{HCOONa}$ (Merck, p.a). and $0.033 \mathrm{M} \mathrm{H}_{2} \mathrm{SO}_{4}$ was employed as electrolyte. The formic acid buffer solution has a constant $\mathrm{pH}$ value of ca. 2.85 and has the resistivity of $107 \Omega \mathrm{cm}$. After the experiments, the WE was treated in conc. $\mathrm{HClO}_{4}$ (Merck, suprapure) and conc. $\mathrm{HNO}_{3}$ (Merck, suprapure) to completely remove bismuth adatom residuals.

\section{Bismuth modifications}

Unlike most other studies of formic acid oxidation on bismuth modified electrodes, bismuth adatom modifications of the Pt WE was done through underpotential deposition (UPD) of bismuth in this experiment. In other words, bismuth ions were present in the bulk electrolyte throughout the entire experiment. A $1 \times 10^{-3} \mathrm{M} \mathrm{Bi}^{3+}$ containing solution was prepared by dissolution of high-purity bismuth(III) oxide $\left(\mathrm{Bi}_{2} \mathrm{O}_{3}\right.$, Strem Chemicals Inc., 99.9998\%) in $0.5 \mathrm{M}$ $\mathrm{HClO}_{4}$. Appropriate amounts of the $1 \times 10^{-3} \mathrm{M} \mathrm{Bi}^{3+}$ solution were added to the electrolyte to obtain final concentrations of $\mathrm{Bi}^{3+}$ ranging from $1 \times 10^{-5}$ to $1 \times 10^{-6}$. After adding bismuth ions and prior to each measurement, the initial
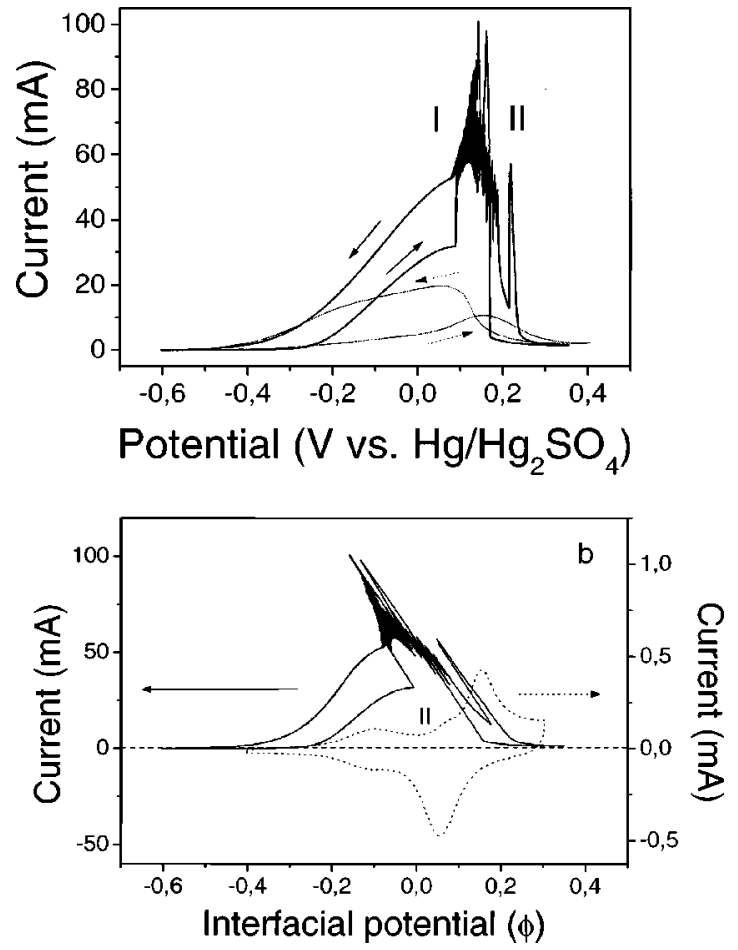

FIG. 1. Cyclic voltammetry of electrocatalytic oxidation of formic acid on Pt electrode. (a) Solid line, $1 \times 10^{-6} \mathrm{M} \mathrm{Bi}^{3+}$; dotted line, without $\mathrm{Bi}^{3+}$. Direct comparison of the voltammetric features on bismuth modified $\mathrm{Pt}$ in bulk solution of $0.5 \mathrm{M} \mathrm{H}_{2} \mathrm{SO}_{4}+0.999 \mathrm{M} \mathrm{NaOH}$ with $1 \times 10^{-6} \mathrm{M} \mathrm{Bi}^{3+}$ $(\mathrm{pH}=$ ca. 3, dotted line) and the oscillatory formic acid oxidation in $0.1 \mathrm{M}$ $\mathrm{HCOONa} / 0.033 \mathrm{M} \mathrm{H}_{2} \mathrm{SO}_{4}$ with $1 \times 10^{-6} \mathrm{M} \mathrm{Bi}^{3+}(\mathrm{pH}=2.8$, solid line $)$ on the scale of the real interfacial (double layer) potential. The plots were obtained after correction for ohmic potential drops across the electrolyte as determined from impedance measurements at large frequencies. Scan rate is $10 \mathrm{mV} / \mathrm{s}$.

WE potential was kept at its open circuit potential (OCP, $-320 \mathrm{mV}$ vs $\mathrm{Hg} / \mathrm{Hg}_{2} \mathrm{SO}_{4}$ ) for 3 min to allow for a stationary initial bismuth coverage on the $\mathrm{Pt}$ electrode.

\section{Impedance spectroscopy}

Impedance spectra were measured at various constant outer potentials using a potentiostat \& galvanostat $(\mathrm{EG} \& \mathrm{G}$, PAR 273A)/log-in amplifier (NF Circuit design Block Co., Frequency response analyzer, Model S-5720) setup attached to the electrochemical cell described earlier. ${ }^{31,32}$ The desired outer applied potentials were adjusted during the anodic voltammetric scan, then the system was allowed to settle to stationary mass-transport conditions. Between two successive measurements, the electrode potential was scanned once between $+250 \mathrm{mV}$ and $-500 \mathrm{mV}$ to ensure comparable surface conditions. The frequency range investigated reached from $10 \mathrm{kHz}$ to $0.1 \mathrm{~Hz}$ with 25 points per decade.

\section{RESULTS}

\section{A. Oscillatory formic acid oxidation on Pt/bismuth UPD}

\section{Cyclic voltammetry (CV)}

Figure 1 illustrates the effect of bismuth UPD on the voltammetry of formic acid oxidation on Pt. With the dotted curve indicating the $\mathrm{CV}$ of the unmodified $\mathrm{Pt}$ ring electrode, 

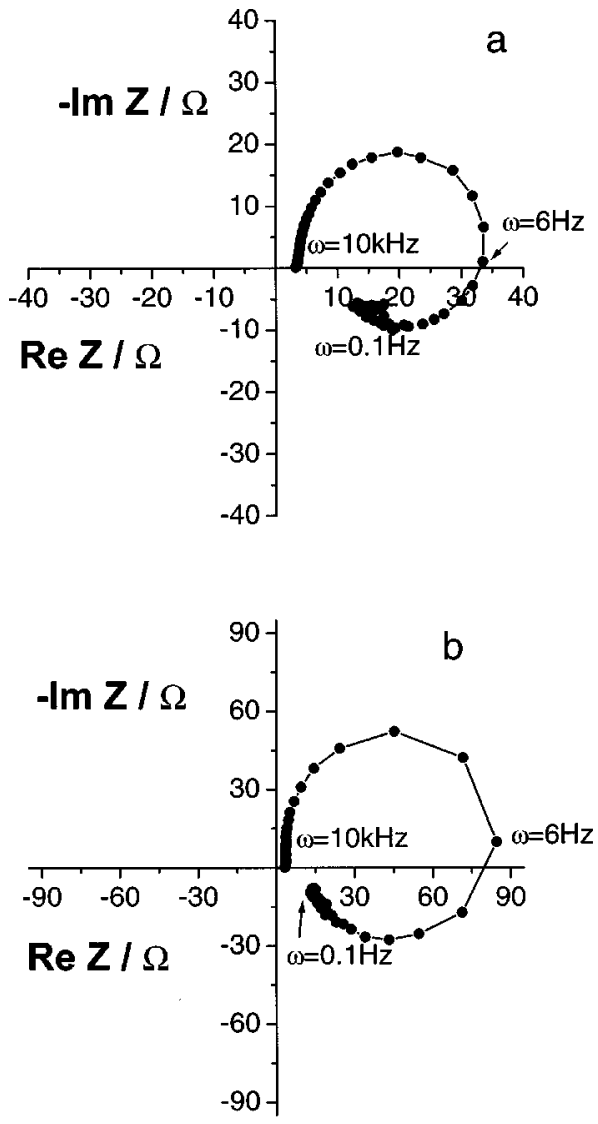
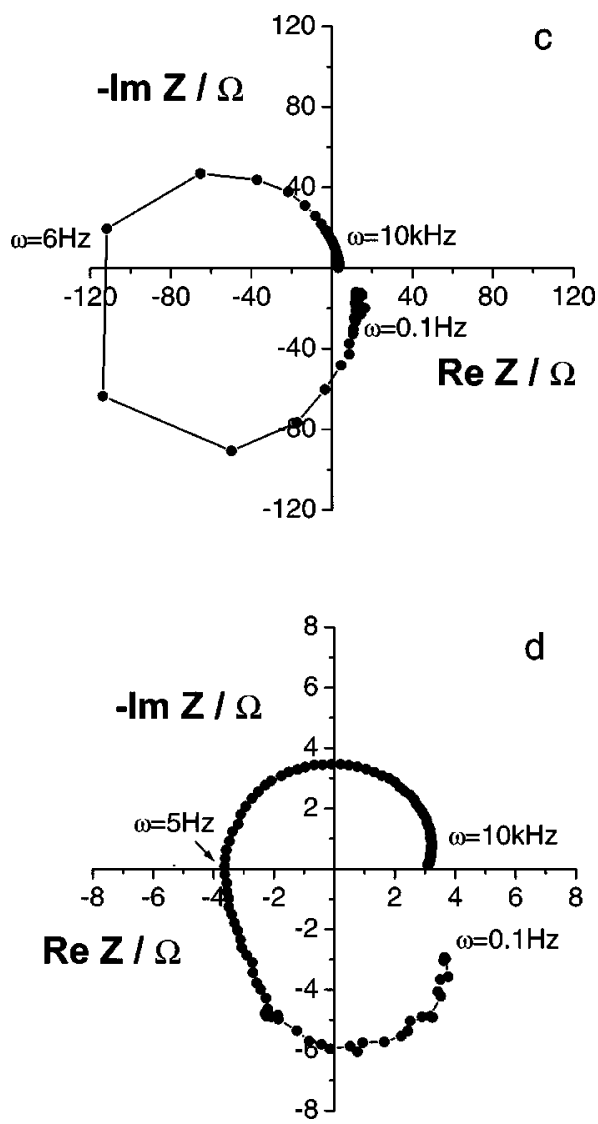

FIG. 2. Impedance behavior of the formic acid oxidation on bismuth modified $\mathrm{Pt}$ at various outer potentials in $0.1 \mathrm{M} \mathrm{HCOONa}_{0} 0.033 \mathrm{M} \mathrm{H} \mathrm{SO}_{4}$ with $1 \times 10^{-6} \mathrm{M} \mathrm{Bi}^{3+}$ : (a) $+60 \mathrm{mV},(\mathrm{b})+70 \mathrm{mV}$, (c) $+80 \mathrm{mV}$, and (d) $+130 \mathrm{mV}$.

the solid line in Fig. 1(a) demonstrates a significant enhancement of the catalytic activity by adding $1 \times 10^{-6} \mathrm{M} \mathrm{Bi}^{3+}$ on the anodic scan. The peak current associated with formic acid oxidation is significantly higher involving a broad potential range of current oscillations between $+90 \mathrm{mV}$ and $+240 \mathrm{mV}$. Though the current tends to be smaller at low voltage, this result is in good agreement with previous studies that the current for formic acid oxidation may increase by up to a factor of $50 .{ }^{33}$ The oscillatory $\mathrm{CV}$ was found to be reproducible for many successive voltammetric cycles.

The parameter dependence of the current oscillations exhibits the following interesting features. Current oscillations with high frequency and small amplitude abruptly set in after an upward current step occurring around the peak maximum on the positive scan. Immediately after the discontinuous onset, the oscillations appear to occur around an unstable stationary $I / U$ curve of positive slope [Fig. 1(a-I)]. In contrast, at higher overpotentials, the oscillatory current spikes follow an $I / U$ profile of negative slope with higher amplitude and smaller frequency, as shown in Fig. 1(a-II). The increase of the concentration of bismuth ion causes the abrupt onset of oscillatory behavior to shift to smaller outer potentials, and also leads to a decrease in the stability and the reproducibility of the oscillatory operating states.

A correlation of oscillatory voltammetry in Fig. 1(a) and bismuth redox features in Fig. 1(b) in $\mathrm{pH} 3$ solution without formic acid may offer valuable clues as to the chemical surface processes that occur under oscillatory conditions. How- ever, a direct comparison of Fig. 1(a) with CV profiles in $\mathrm{pH} 3$ solution without formic acid on bismuth-UPD Pt [Fig. 1(b), dotted line) is not possible due to the uncompensated solution resistance. Thus, corrected for the ohmic potential drop ( $\left(\mathrm{R}_{\text {solution }}\right)$ the curve plotted as solid line in Fig. 1(b) refers to the true interfacial potential. In Fig. 1(b) $\left(1 \times 10^{-6} \mathrm{M} \mathrm{Bi}^{3+}\right)$, one recognizes the current step to occur near the cathodic onset of the second plateau (II), which marks the beginning of $\mathrm{Bi}$ oxidation on the surface.

\section{Impedance spectroscopy}

To analyze the origin of the current oscillations in Fig. 1(a), impedance spectra were measured for various potentiostatic operation points prior to the onset of periodic instabilities. Nyquist plots at four different applied potentials $U$ are shown in Fig. 2. At $U=+60 \mathrm{mV}$ and $U=+70 \mathrm{mV}$ [Figs. 2(a) and 2(b)], the impedance plot $Z(\omega)$ qualitatively exhibits a clockwise capacitive-inductive loop indicating dynamically stable stationary operating points. At $+70 \mathrm{mV}$, the intersection of the impedance plot with the real axis occurs at higher ohmic resistances compared to Fig. 2(a) suggesting a gradual blow-up of the Nyquist loop for increasing $U$. The impedance plot is drastically changed at $+80 \mathrm{mV}$ in Fig. 2(c). $Z(\omega)$ wraps around the origin counter-clockwise, that is, there is an intersection between the real axis and $Z(\omega)$ where $Z$ takes on a (negative) purely real value at some finite frequency $\omega_{0}=4-6 . Z(\omega)$ again exhibits positive real resis- 

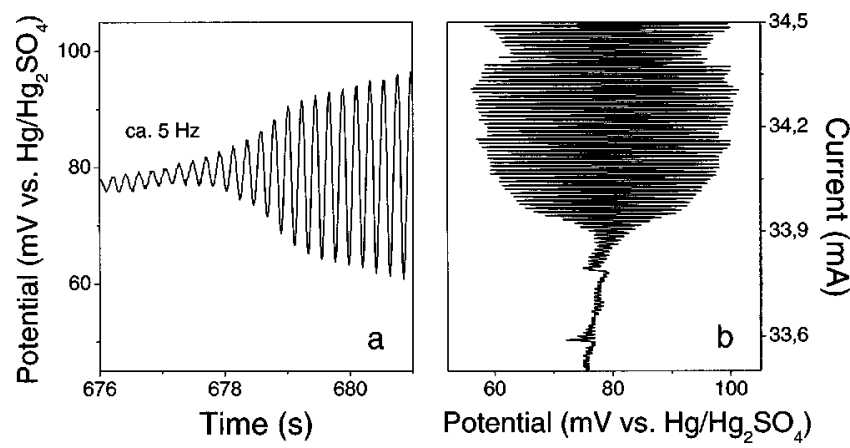

FIG. 3. Onset of sustained potential oscillations (Hopf bifurcation) in the galvanostatic operation mode with scan rate of $0.05 \mathrm{~mA} / \mathrm{s}$. (a) $I / \phi$ plot, (b) $\phi / t$ plot. Bulk solutions and parameters as in Fig. 2.

tances for very low frequencies and this qualitative shape of the Nyquist plot in Fig. 2(d) is continuous towards higher values $U$ of $+130 \mathrm{mV}$.

The detailed shape of the Nyquist plot bears information on the mechanistic origin of the oscillatory behavior, which can be used to predict the dynamical stability of oscillatory systems. The significance of the distinct value $\omega_{0}$ in Fig. 2(c) where $\operatorname{Im} Z=0$ with $\operatorname{Re} Z<0$ becomes clear in Fig. 3. A galvanostatic scan of the formic acid oxidation, which is shown in Fig. 3(a) ( $\phi / t$ plot) and Fig. 3(b) ( $I / \phi$ plot), reveals an onset potential of sustained oscillations between $+70 \mathrm{mV}$ and $+80 \mathrm{mV}$, with an initial oscillation frequency of 4-6 Hz. As discussed in previous work, ${ }^{26,34,35}$ the critical potential, where the Nyquist plot blows up such that $\operatorname{Re} Z\left(\omega_{0}\right)$ approaches \pm infinity, marks the galvanostatic transition to periodic behavior, i.e., Hopf bifurcation. The value of $\omega_{0}$ represents the intrinsic frequency of the oscilla- tion in the electrocatalytic oxidation of $\mathrm{HCOOH}$ on Pt modified by bismuth.

\section{B. Spatial patterns in the interfacial potential}

This section deals with spatio-temporal distributions of the local electrode potential underlying the oscillatory instability. Due to negligible solution resistance between potential microprobe and WE, the local potential essentially reflects the interfacial electrode potential. For a better understanding of spatio-temporal pattern formation recorded by the interfacial potential near the WE electrode, we introduce the experimental measurement [Fig. 4(a)] of a typical space-time plot of the local potential along the Pt ring electrode when constant potential is applied within the oscillatory range of Fig. 1(a). According to $U=\mathrm{IR}+\phi$, higher potentials correspond to low local catalytic activity caused by poisoning, while low values of interfacial potentials make a large contribution to the total current due to depoisoning phenomena. Figure 4(a) shows that a narrow domain of high activity (blue) is continuously traveling around the otherwise passive ring surface, which is called a traveling activation pulse. A theoretical simulation of a traveling pulse is shown in Fig. 4(b) (for the model used see Refs. 20, 36, and 37 and discussion).

\section{Pattern sequences on the cyclic voltammogram}

Figure 5(a) shows the temporal signal of current oscillations during a cyclic voltammogram with a scan rate of 5 $\mathrm{mV} / \mathrm{s}$. The behavior in the time domain reveals a transition from harmonic large-amplitude oscillations to a complex pattern of smaller peaks. The spatial patterns underlying the anodic scan of Fig. 5(a) are reported in Figs. 5(b)-5(d). At the beginning of oscillations, the potential distribution in Fig. 5(b) clearly represents standing waves (SW), while traveling
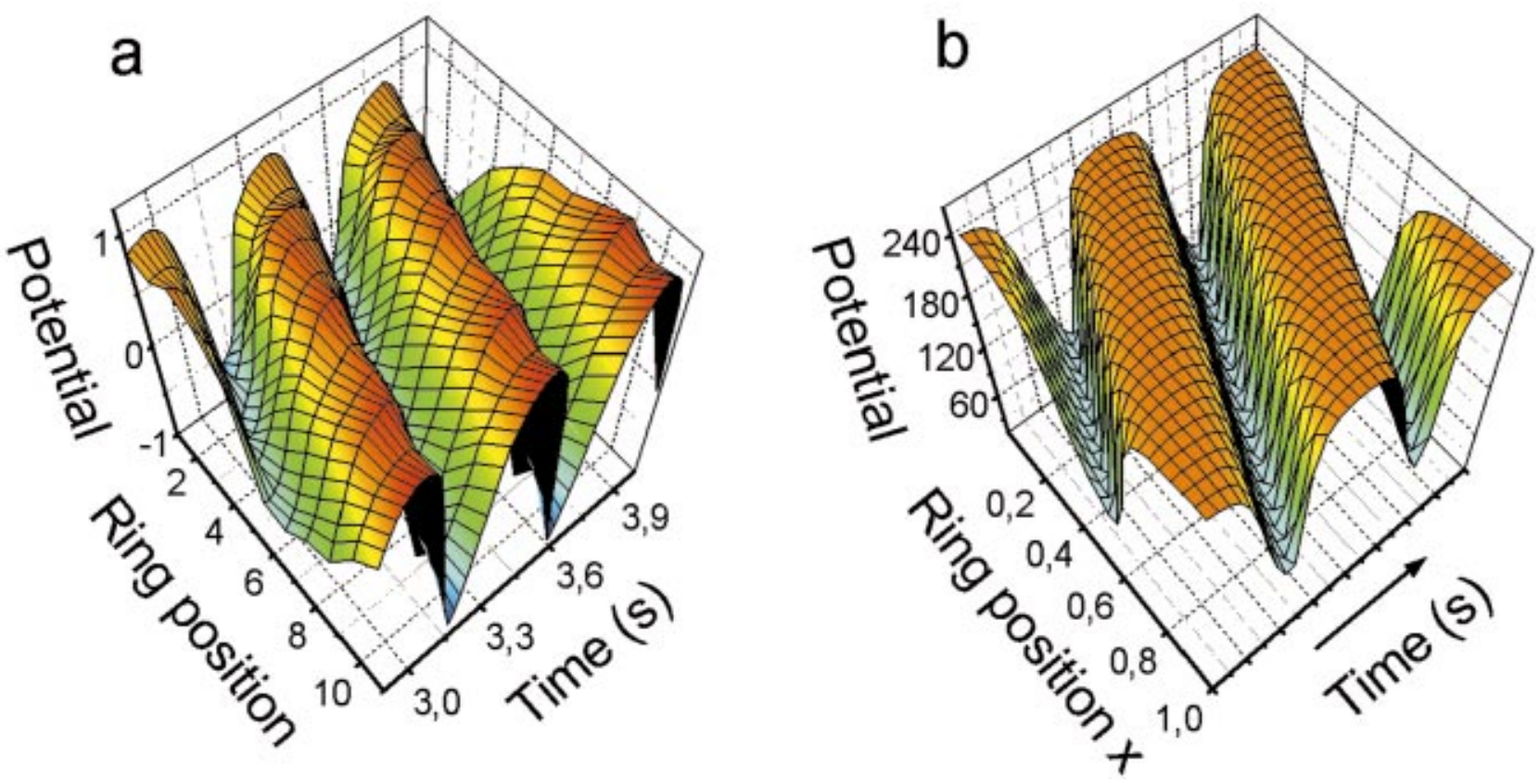

FIG. 4. (Color) Spatio-temporal distribution of the local interfacial potential during current oscillations at constant outer potential $U$ (traveling catalytic pulse).

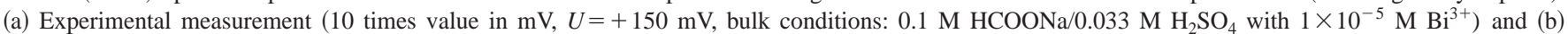
theoretical simulation of traveling pulse (for model details see Refs. 36 and 46). 

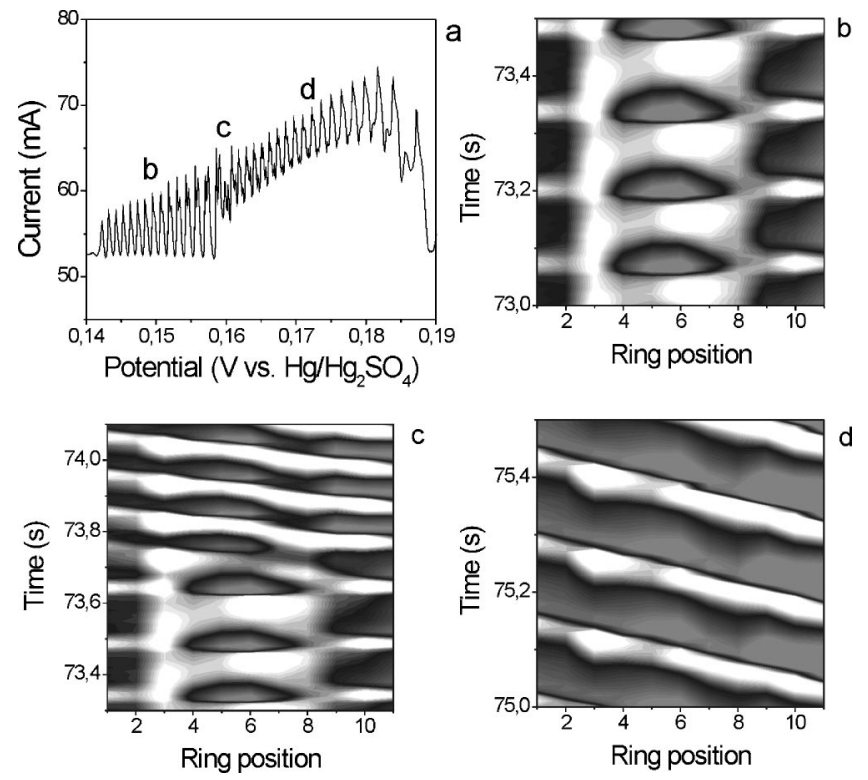

FIG. 5. (a) Oscillatory current/potential profile during slow anodic scanning. The change in wave form near $t=74.5 \mathrm{~s}$ indicates changes in the spatial dynamics. (b)-(d) Spatio-temporal profile of the interfacial potential corresponding to (a). (b) Oscillatory standing wave regime, (c) pattern transformation from SW to Pulse, and (d) traveling catalytic pulses. Scan rate is 5 $\mathrm{mV} / \mathrm{s}$ and electrolyte is $0.1 \mathrm{M} \mathrm{HCOONa} / 0.033 \mathrm{M} \mathrm{H}_{2} \mathrm{SO}_{4}$ with $5 \times 10^{-7} \mathrm{M}$ $\mathrm{Bi}^{3+}$.

pulses prevail at higher overpotentials [Fig. 5(d)]. When reversing the anodic scan within a pulse regime, the pulse velocity first becomes more and more inhomogeneous along the ring. Then, the spatio-temporal dynamics passed through the transition state, before it comes back to SW. Oscillatory standing waves (SW) were recently described in the electrocatalytic oxidation of formic acid on unmodified $\mathrm{Pt}$ electrodes. ${ }^{23}$ It is characterized by the sustained periodic alternation of an active (low interfacial potentials) and a passive (high interfacial potentials) domain in space-time without spatial propagation.

\section{Pattern sequences at fixed applied potentials}

Figure 6(a) depicts the time-periodic evolution of the total current when keeping the applied potential $U$ in the middle of the oscillatory range on the anodic scan. First, the time average of the integral current slowly decreases exhibiting a complex oscillatory regime of relatively small amplitude. Then, after some time, the shape of the oscillations suddenly undergoes a distinctive transformation to a period-2 regime with larger amplitude. Figures 6(b)-6(d) show the corresponding processes in the local electrode potentials. The initial dynamics represent traveling catalytic pulses as shown in Fig. 6(b). At the transition, the pulse stops, giving way to a dynamic regime resembling oscillatory standing waves [Fig. 6(c)] which subsequently remain stable for long observation times [Fig. 6(d)]. [However, these standing waves do not seem to arise from the same bifurcation as in Fig. 5; most likely they are caused by propagation failure of pulses due to surface inhomogeneities.]

Without forced mass transport, high-amplitude current oscillations at fixed $U$ observed in Fig. 6 usually entail
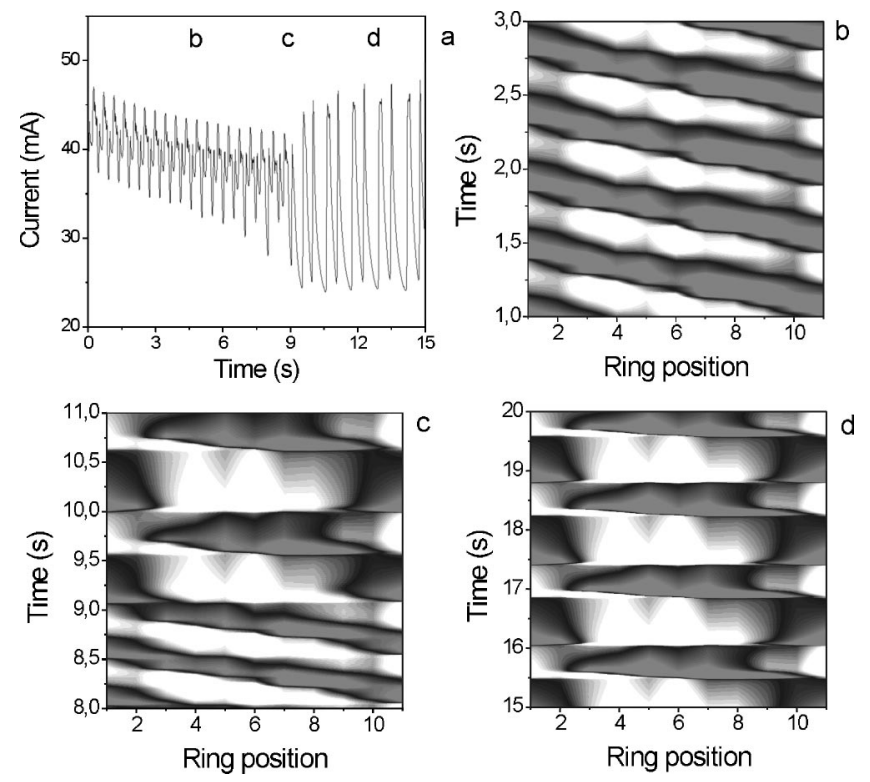

FIG. 6. (a) Sustained current oscillations at constant applied potential $U$. A qualitative change in the oscillatory wave form (around $t=9 \mathrm{~s}$ ) indicates changes in the spatial potential distribution. (b)-(d) Spatio-temporal profile of the interfacial potential corresponding to the time series (b)-(d) in (a). (b) Traveling catalytic pulses, (c) propagation failure of pulses, and (d) final oscillatory standing wave regime most likely due to defects blocking transition of pulses. Experimental conditions: constant $U=+152 \mathrm{mV}, 0.1 \mathrm{M}$ $\mathrm{HCOONa} / 0.033 \mathrm{M} \mathrm{H}_{2} \mathrm{SO}_{4}, 1 \times 10^{-6} \mathrm{M} \mathrm{Bi}^{3+}$.

gradual depletion of dynamical volume species such as the local concentration of an electroactive species at the electrode. But driven into the mass-transport-limited regime, the dynamics of the system may significantly be altered. In order to investigate effects of mass transport on the pattern forming processes, $\mathrm{N}_{2}$ bubbling was applied, as shown in Fig. 7. Reproducible pattern formation, standing waves (SW), traveling pulse (Pulse), and defect mediated standing waves (DSW), are sequentially observed by repetitive restoring of the original mass-transport conditions, i.e., repeated replenishment of the species near the surface through forced con-

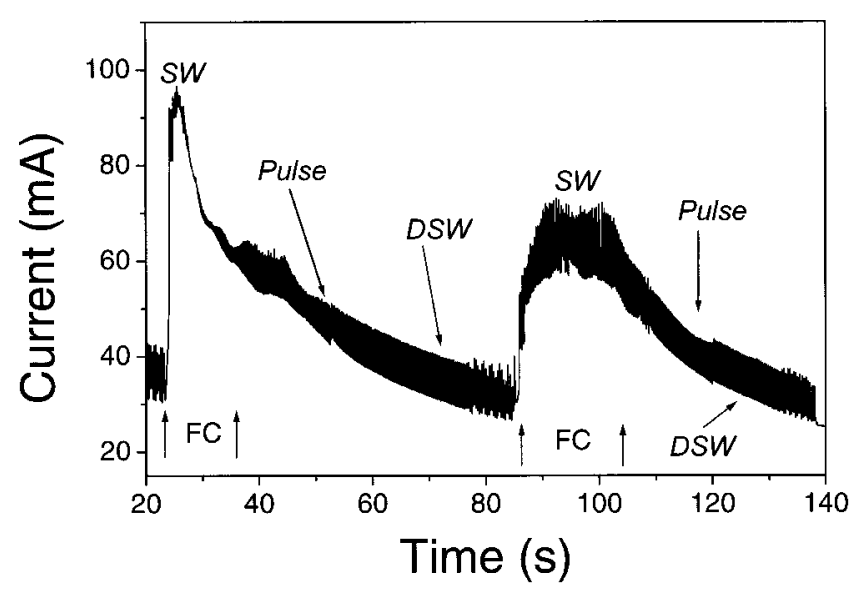

FIG. 7. Effect of forced convection on the current oscillations and the corresponding potential patterns at constant outer potential. Experimental conditions as in Fig. 6. [Forced convection (FC), standing waves (SW), traveling pulse (Pulse), defect mediated standing waves (DSW).] 


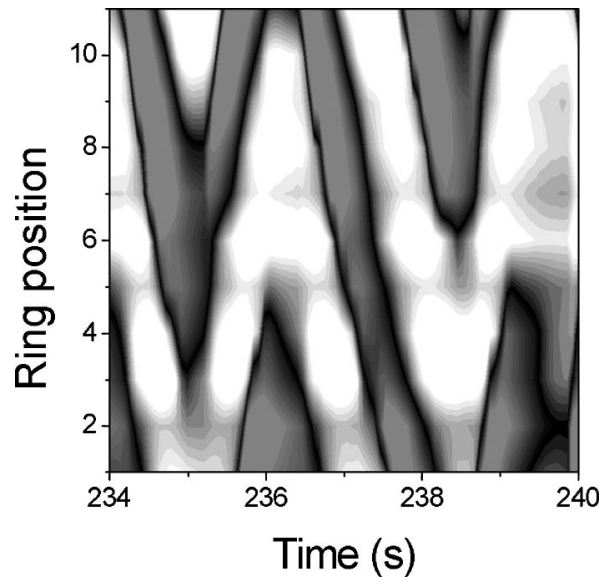

FIG. 8. Defect-mediated reversal of direction of traveling catalytic pulses at fixed outer potential (scan rate $=10 \mathrm{mV} / \mathrm{s}, 0.1 \mathrm{M} \mathrm{HCOONa} / 0.033 \mathrm{M} \mathrm{H}_{2} \mathrm{SO}_{4}$ with $5 \times 10^{-7} \mathrm{M} \mathrm{Bi}^{3+}$ ).

vection. Obviously, the important parameter for the observation of the spatio-temporal patterns is the local concentration drift of the species near the electrode.

To fully understand the organizing mechanisms of the transitions between distinct potential patterns (bifurcation), aside from controllable parameter (system constraints) such as bulk concentrations or applied potential, the organizing role of surface points with slightly varying catalytic properties (surface defects) must be considered. While changes in constraints are sufficient to organize bifurcation when one pattern becomes unstable in favor of another, defects continuously act as perturbations and may mediate between two coexisting stable dynamical states when their perturbing effect becomes sufficiently strong. Hence, an experimentally observed bifurcation can be due to the combined effect of changes in control parameters and the action of defects. Figure 8 shows a space-time plot of a traveling pulse associated with oscillatory currents at fixed potential $U$. Approaching the ring position $2-4$ at time $t=234-235 \mathrm{~s}$, the pulse spontaneously stops short near ring position $3-4$, followed by a reversal of its direction. Subsequently, the pulse reversal repeats close to the same locations when approached from the opposite direction.

Obviously, electrode positions at 2-4 are more difficult to activate by a passing activation pulse and, therefore, may constitute a barrier for pulses with sufficiently low amplitude. If this occurs, the dynamic response of the system to the perturbation by the defect is the spontaneous transition to the (ideally equally stable) coexisting traveling pulse in the opposite direction.

\section{DISCUSSION}

Impedance spectroscopy showed that formic acid oxidation in the presence of $\mathrm{Bi}^{3+}$ is still an HNDR oscillator (hidden negative differential resistance). The negative resistance is (as in the absence of $\mathrm{Bi}^{3+}$ ) due to site blocking by $\mathrm{OH}$ adsorption. ${ }^{20}$ Adsorbed Bi largely suppresses the formation of $\mathrm{CO}$ on the electrode, which explains the significantly higher currents. The positive impedance on a slower time scale may nevertheless still be due to (some) CO formation, but here increasing $\mathrm{Bi}$ coverage at low overpotentials may also have a site-blocking effect.

The electrocatalyzed oxidation of formic acid on $\mathrm{Pt}$ modified by bismuth spontaneously undergoes transitions between different dynamic states, from homogeneous catalytic activity to states characterized by a spatio-temporally inhomogeneous distribution of the interfacial electrode potential (dynamical electrocatalytic potential patterns). The prevailing space-time patterns observed under the used ring electrode geometry have been oscillatory standing waves ${ }^{23,37}$ as well as traveling electrocatalytic pulses, both associated with sustained oscillations in the total current. The patterns and the oscillations strongly depend on external control parameters such as the concentration of the solution and the applied potential.

\section{A. Spatio-temporal patterns and the effect of surface defects}

Inhomogeneous distributions of the local potential at the electrified interface occurred around the maximum of the oxidation rate. Figure 4(a) experimentally demonstrates, for the first time, traveling activation pulses in an electrocatalytic reaction. In electrochemistry, traveling pulses were so far reported only during metal dissolution of Ni (Refs. 38 and 39) and Co in acidic media. ${ }^{36,40-42}$ Aside from electrochemistry, traveling pulse dynamics can also occur in heterogeneous gas-phase catalysis under both isothermal ${ }^{43}$ and thermokinetic conditions. ${ }^{44}$

An activation pulse is a traveling domain of high catalytic activity invading a passive region of the electrode. Thus, for periodic boundary conditions (ring electrode), each location on the WE becomes repeatedly reignited, and thus a stationary homogeneous deactivation of the entire catalytic interface is prevented. Ideally, pulses with a fixed domain size should yield a constant integral current signal for homogeneous system parameters. As shown in Figs. 4, 5, and 6, however, surface inhomogeneities may constantly deform the pulse along the ring resulting in an oscillatory integral current signal. The second interfacial potential pattern prevailing under the parameters chosen is oscillatory standing waves. These regimes involve spatially and temporally alternating domains of higher and lower activities with two fixed points of the electrode (nodes) of constant catalytic activity. Unlike metal dissolution reactions, ${ }^{45}$ standing waves were found to be the only ordered pattern in the electrocatalyzed oxidation of formic acid on pure Pt electrodes. ${ }^{23}$ The observed correlation between space-time dynamics and external system parameters such as concentrations and outer potential of $U$ suggests a convenient strategy to control interfacial potential distributions in the electrocatalytic process in question.

Recently, a reaction-migration formalism introduced by Christoph $^{36,46}$ presented a realistic theoretical and numerical description of pattern formation in electrochemistry. The distinctive approach of the model allowed the theoretical prediction of inhomogeneous patterns even under complicated electrode geometry. Assuming the validity of the Laplace equation within the electrolyte, potential theory leads to an 


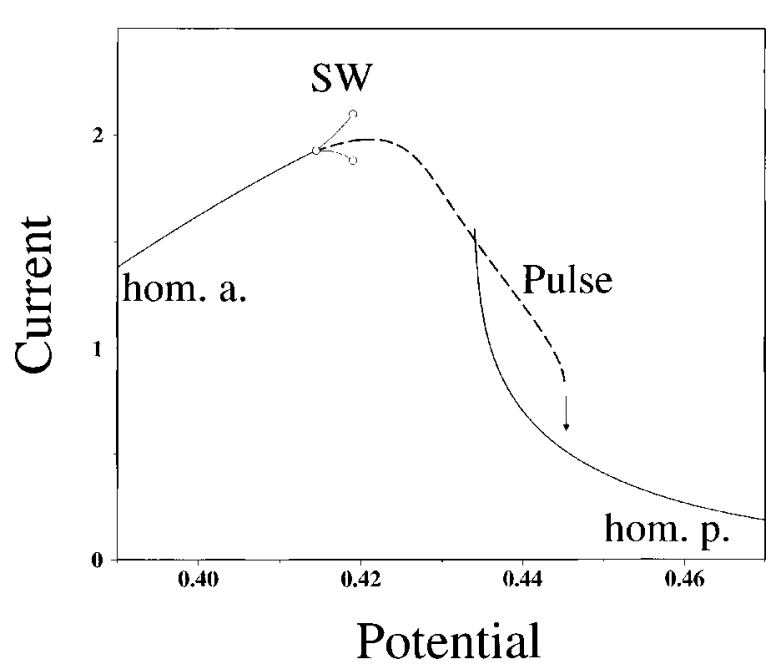

FIG. 9. Numerical bifurcation diagram illustrating stable spatio-temporal patterns of the double layer potential in dependence of the applied potential. For dynamic regimes that are associated with stationary integral migration currents (hom.a., hom.p., and Pulses), $I$ is plotted vs $U$. In case of patterns standing wave patterns SW, which are associated with time-periodic currents, the current minimum and maximum are plotted on the $y$ axis (solid line, hom.a. $=$ homogeneous active stationary state; hom.p. $=$ homogeneous passive stationary state; open circles, SW=standing waves; dashed line, Pulse $=$ traveling pulses). The diagram was calculated using a dimensionless HNDR model. Parameters were chosen such that the one-parameter diagram cut through the oscillatory regime.

integro-differential equation for the description of the evolution of the interfacial (double layer) potential $\phi$ across the working electrode. $^{23,42}$ The equation for $\phi$ consisted of (i) local faradaic reaction terms describing the detailed chemistry of the reaction, (ii) the local migration currents, and (iii) an integral term accounting for the spatial migration coupling parallel to the electrified interface. The integral involved a spatial coupling function $H$ indicating the sign and strength of the coupling between two different points on the electrode. The qualitative shape of $H$ was found to be critically dependent on the geometry of RE and WE. For the electrode geometry chosen, a ring type electrode, $H$ revealed a negative coupling of the interfacial potential between opposite points of the ring (negative long-range coupling), while adjacent points were coupled positively (positive short-range coupling). Negative coupling generally leads to a desynchronization of the interfacial potential, and therefore generally increases the tendency of an electrochemical system towards the formation of inhomogeneous interfacial patterns. The mechanism used was a generic HNDR oscillator, including two chemical species, one of which adsorbs at lower and desorbs at higher potential (giving rise to a positive differential resistance); the other adsorbs (desorbs) at higher (lower) potential (causing a negative differential resistance). The latter is due to site blocking by $\mathrm{OH}$ adsorption in the present experimental system, while the former most likely corresponds to adsorbed $\mathrm{CO}$ and/or Bi.

Figure 9 shows the theoretical model predictions concerning the patterns in the interfacial potentials in dependence of the fixed applied outer potential. The bifurcation diagram was obtained for parameters and electrode geometry comparable to the present experiments, that is, under condi- tions of negative long-range coupling. For an illustrative explanation of the various regimes, a mode picture will be invoked. With increasing potential, the homogeneous active steady state, hom.a., loses stability in favor of the first inhomogeneous mode consistent with the periodic boundary conditions of the ring. The space-time amplitude $A$ of the resulting oscillatory standing waves (SW) is governed by a term like $A \approx a(t) \sin (2 \pi / L \cdot x)$, where $L$ and $x$ are the circumference of the ring electrode and the spatial coordinate along the ring, respectively. The function $a(t)$ denotes the timedependent amplitude of the standing wave. At higher values of the applied potential $U$, the amplitude $a(t)$ of the active first mode strongly increases continuously exciting the corresponding, phase-shifted cosine mode by means of highermode coupling. ${ }^{46}$ With one or more sine and cosine terms suitably superimposed, SW transforms into pulse patterns at ca. +420 , which remain stable up to high values of $U$. Since either pulse direction is equistable in the model calculations, the resulting pulse direction is entirely determined by the initial conditions. Transitions between directions may only be achieved by perturbations (see Fig. 8). Finally, at high $U$, the system jumps to the homogeneous passive steady state. Reversing the potential scan, Fig. 9 predicts the system to jump back to traveling pulses. In the simulations the pulse pattern remains stable up to the transition to the homogeneous active steady state. Generally one-dimensional target patterns can coexist with traveling pulses near the homogeneous passive state (hom.p.), provided the difference in time scales of the variables is sufficiently large. ${ }^{46}$ No target patterns are observed in the present experiments, but they have been reported previously in metal dissolution system. ${ }^{36}$

While the predicted bifurcation sequence of Fig. 9 on the anodic scan favorably compare with experimental results in Fig. 5, the observed spontaneous transition from pulses to SW on the negative-going scan seems to contradict the model. However, recall that Fig. 9 represents the behavior of an ideal reactive system with no spatial inhomogeneities in the parameters. In contrast, considering the measured pulse dynamics in Fig. 5, the experimental electrode surface does exhibit points of varying catalytic activity (surface defects). If sufficiently strong, defects may mediate the transition between coexisting regimes, for instance, in Fig. 8 between regimes with opposite pulse direction. Scanning in the negative direction within the pulse regime, the amplitudes of the individual superimposed modes slowly decrease. At some critical point, surface defects may be sufficient to suppress one (or more) of the inhomogeneous modes, for instance, if a passive defect happens to be near a point of high amplitude. In the simplest case of a traveling pulse made up by one sine and cosine mode, suppressing either of them leads to a reappearance of SW.

A similar line of argument can be invoked for the transition from pulses to DSW in Figs. 6 and 7. In the course of current oscillations at fixed applied potential, the average solution concentration of formic acid near the electrode surface, $c_{\mathrm{FA}}$, gradually decreases with time. This amounts to a constant drift in a control parameter driving the system slowly towards the stationary passive regime. Near the tran- 
sition, the amplitude of the pulse decreases making it more susceptible to the influence of surface defects. Finally, defects will give rise to nonrotating patterns, which may appear very similar to the initial standing waves, but are of different physical origin and do not arise for the same bifurcation scenario.

\section{CONCLUSIONS}

The electrocatalytic oxidation of formic acid on bismuth modified Pt can be operated at parameters where the local interfacial potential, and therefore the reaction rate, exhibits spatio-temporally inhomogeneous distributions. The origin of the patterns lies in the combined effects of the nonlinear chemistry involved in formic acid oxidation and the negative long-range coupling of the interfacial potential due to the chosen geometry. Due to the sustained periodic reactivation of certain parts of the catalytic interface, both electrocatalytic pulses and oscillatory standing waves impede complete homogeneous deactivation of the catalytic surface. This generally results in a prolonged catalytic reactivity of the electrode as compared to stationary operation conditions.

\section{ACKNOWLEDGMENTS}

The authors are deeply indebted to C. Eickes for his technical assistance with impedance spectroscopy. Part of this work was supported by the Deutsche Forschungsgemeinschaft (DFG) under SFB 555.

${ }^{1}$ W. Vielstich, Brennstoffelemente (Weinheim, Berlin, 1965).

${ }^{2}$ M. W. Breiter, in Anorganische und Allgemeine Chemie in Einzeldarstellungen Band IX (Springer, New York, 1968).

${ }^{3}$ W. Vielstich, Fuel Cells (Wiley, New York, 1970).

${ }^{4}$ R. Parsons and T. VanderNoot, J. Electroanal. Chem. 257, 9 (1988).

${ }^{5}$ J. Lipkoski and P. N. Ross, Electrocatalysis (Wiley-VCH, New York, 1998).

${ }^{6} \mathrm{~S}$. Gottesfeld, in Advances in Electrochemical Science and Engineering, edited by R. C. Alkire, H. Gerischer, D. M. Kolb, and C. W. Tobias (Wiley-VCH, New York, 1997), Vol. 5.

${ }^{7}$ S. Wasmus and A. Küver, J. Electroanal. Chem. 461, 14 (1999).

${ }^{8}$ E. Müller, Z. Elektrochem. 28, 101 (1922).

${ }^{9}$ E. Müller, Z. Elektrochem. 33, 209 (1927).

${ }^{10}$ E. Müller and S. Tanaka, Z. Elektrochem. 34, 256 (1928).

${ }^{11}$ J. Wojtowicz, N. Marincic, and B. E. Conway, J. Chem. Phys. 48, 4333 (1968).

${ }^{12}$ M. Schell, F. N. Albahadily, J. Safar, and Y. Xu, J. Phys. Chem. 90, 813 (1989).
${ }^{13}$ M. Schell, J. Electroanal. Chem. 457, 221 (1998).

${ }^{14}$ M. Hachkar, B. Beden, and C. Lamy, J. Electroanal. Chem. 287, 81 (1990).

${ }^{15}$ Y. Xu and M. Schell, J. Phys. Chem. 94, 7137 (1990).

${ }^{16}$ F. N. Albahadily and M. Schell, J. Electroanal. Chem. 308, 151 (1991).

${ }^{17}$ X. Cai and M. Schell, Electrochim. Acta 37, 673 (1992).

${ }^{18}$ H. Okamoto and N. Tanaka, Electrochim. Acta 38, 503 (1993).

${ }^{19}$ M. Krausa and W. Vielstich, J. Electroanal. Chem. 399, 7 (1995).

${ }^{20}$ P. Strasser, M. Lübke, F. Raspel, M. Eiswirth, and G. Ertl, J. Chem. Phys. 107, 979 (1997).

${ }^{21}$ H. Okamoto, N. Tanaka, and M. Naito, J. Phys. Chem. A 101, 8480 (1997).

${ }^{22}$ J. Christoph, P. Strasser, M. Eiswirth, and G. Ertl, Science 284, 291 (1999).

${ }^{23}$ P. Strasser, J. Christoph, W.-F. Lin, M. Eiswirth, and J. L. Hudson, J. Phys. Chem. A 104, 1854 (2000).

${ }^{24}$ M. T. M. Koper, Electrochim. Acta 37, 1771 (1992).

${ }^{25}$ M. T. M. Koper and J. H. Sluyters, J. Electroanal. Chem. 371, 149 (1994).

${ }^{26}$ M. T. M. Koper, Adv. Chem. Phys. 92, 161 (1996).

${ }^{27} \mathrm{~K}$. Krischer, in Modern Aspects in Electrochemistry 32, edited by J. O. Bockris, B. E. Conway, and R. E. White (Plenum, New York, 1999).

${ }^{28}$ J. L. Hudson and T. T. Tsotsis, Chem. Eng. Sci. 49, 1493 (1994).

${ }^{29}$ S. Shigang and L. Yan, Prog. Nat. Sci. 2, 351 (1992).

${ }^{30}$ S. Smith, K. Ben-Dor, and H. Abruna, Langmuir 15, 7325 (1999).

${ }^{31}$ P. Strasser, M. Lübke, P. Parmanada, M. Eiswirth, and G. Ertl, J. Phys. Chem. 102, 3227 (1998).

${ }^{32}$ C. Eickes, K. G. Weil, and K. Doblhofer, Phys. Chem. Chem. Phys. 2, 5691 (2000).

${ }^{33}$ J. Lipkowski, Z. Shi, A. Chen, B. Pettinger, and C. Bilger, Electrochim. Acta 43, 2875 (1998).

${ }^{34}$ M. T. M. Koper, J. Electroanal. Chem. 409, 175 (1996).

${ }^{35}$ P. Strasser, M. Eiswirth, and M. T. M. Koper, J. Electroanal. Chem. 478, 50 (1999).

${ }^{36}$ J. Christoph, R. Otterstedt, M. Eiswirth, N. Jaeger, and J. Hudson, J. Chem. Phys. 110, 8614 (1999).

${ }^{37}$ P. Strasser, Ph.D. thesis, Freie Universität Berlin, Berlin, 1999 (http:// www.diss.fu-berlin.de/1999/25).

${ }^{38}$ O. Lev, M. Sheintuch, L. Pismen, and C. Yarnitzky, Nature (London) 336, 488 (1988).

${ }^{39}$ O. Lev, M. Sheintuch, H. Yarnitzky, and L. Pismen, Chem. Eng. Sci. 45, 839 (1990).

${ }^{40}$ R. Otterstedt, P. Plath, N. Jaeger, and J. Hudson, Chem. Eng. Sci. 51, 1747 (1996).

${ }^{41}$ R. Otterstedt, P. Plath, N. Jaeger, and J. Hudson, Phys. Rev. E 54, 3744 (1996).

${ }^{42}$ R. Otterstedt, P. Plath, N. Jaeger, and J. Hudson, J. Chem. Soc., Faraday Trans. 92, 2933 (1996).

${ }^{43}$ M. Eiswirth, M. Bär, and H. Rotermund, Physica D 84, 40 (1995).

${ }^{44}$ M. Graham, S. Lane, and D. Luss, J. Phys. Chem. 97, 7564 (1993).

${ }^{45}$ J. Sayer and J. Hudson, Ind. Eng. Chem. Res. 34, 3246 (1995).

${ }^{46}$ J. Christoph. Ph.D. thesis, Freie Universität Berlin, Berlin, 1999 (http:// www.diss.fu-berlin.de/2000/11). 